ARTÍCULO ORIGINAL

\title{
SISTEMA AUTOMATIZADO DE CONTROL TRIBUTARIO, EN LOS GOBIERNOS LOCALES
}

\section{AUTOMATED TAX-CONTROL SYSTEM, IN LOCAL GOVERNMENTS}

\author{
Víctor Adolfo Rodríguez Mateo \\ Universidad Nacional Mayor de San Marcos \\ Magister en Contabilidad de Gestióny Contador Público Colegiado \\ Correo : rbhvrm@gmail.com
}

[Recibido: 19/09/2017 Aceptado: 15/04/2018]

\section{RESUMEN}

La administración municipal en el Perú, es parte de la política nacional de modernización de la gestión pública al 2021. Se conoce de la SUNAT, RENIEC, ONPE y las SAT, sus resultados de gestiones eficientes, el problema es que existen municipalidades con sistemas de administración tributaria muy limitados en sus funciones. Y el "Sistema Automatizado de Control de Valores Tributarios (SACVT)", es una solución presentado en la investigación realizada, de tipo descriptivo y explicativo con el objetivo de, determinar la manera en que influiría la aplicación del sistema, en la gestión administrativa tributaria de la Municipalidad Distrital de San Luis, fuente de la investigación. Para el cual se realizó un análisis correlativo transversal y se aplicaron encuestas dirigidas a dilucidar a las variables analizadas como es, el Sistema Automatizado y la Gestión Administrativa Tributaria, aplicado a los funcionarios y técnicos relacionados con la gerencia de rentas de la Municipalidad. Y el resultado obtenido, confirmó la hipótesis, que el impacto de la aplicación de dicho sistema, es significativo para una mejor gestión tributaria municipal. Considerando que la administración municipal en el Perú es parte de la política nacional de modernización de la gestión pública al 2021 y conociendo la eficiencia de los sistemas de la SUNAT, RENIEC, ONPE y las SAT; la investigación ha permitido plantear soluciones para los sistemas automatizados deficientes en la municipalidad.

Palabras claves : Sistema automatizado, Gestión tributaria, información contable, Recaudación tributaria

\begin{abstract}
The municipal administration in Peru is part of the national policy of modernization of public management to 2021. The SUNAT, RENIEC, ONPE and SAT are known by their efficient management results. The problem is that there are municipalities with tax-administration systems that are very limited in their functions. The "Automated System of Control of Tax Values (SACVT)" is a solution presented in this research, of descriptive and explanatory type with the objective of determining the way in which the application of this system would influence the administrative tax management of the District Municipality of San Luis, source of the investigation. For which a transversal correlative analysis was carried out and surveys were applied to elucidate the analyzed variables such as the Automated System and the Administrative Tax Administration. This was applied to the officials and technicians related to the income management of the Municipality. The result obtained, confirmed the hypothesis, that the impact of the system application is significant for a better municipal tax management. Considering that the municipal administration in Peru is part of the national policy of modernization of public management to 2021, and knowing the efficiency of the systems of SUNAT, RENIEC, ONPE and SAT, the research proposed solutions for the deficient automated systems in the municipality.
\end{abstract}

Keywords: Automated system, tax management, accounting information, tax collection.

\section{INTRODUCCIÓN}

Siendo la información, vital para la toma de decisiones en la gestión pública y privada, la misma que debe ser real y reportada en el momento opor- tuno, hace necesario la aplicación de las tecnologías de la información y la comunicación (TIC), establecidos desde enero del 2002, con la Ley $\mathrm{N}^{\circ}$ 27658, "Ley Marco de Modernización de la Gestión del Estado", en el 2011 se da, el Decreto Supremo Nº 054-2011PCM, "Plan Bicentenario el Perú al 2021" y con el Decreto Supremo N ${ }^{\circ}$ 004-2013-PCM 2013, la "Política de Modernización de la Gestión Pública”, fijando como ejes y pilares de la

(C Los autores. Este artículo es publicado por la Revista Quipukamayoc, Universidad Nacional Mayor de San Marcos. Este es un artículo de acceso abierto, distribuido bajo los términos de la Licencia Creative Commons Atribución-NoComercial-Compartirlgual 4.0 Internacional.(http://creativecommons.org/licenses/by-nc-sa/4.0/), que permite el uso no comercial, distribución y reproducción en cualquier medio, siempre que la obra original sea debidamente citadas. 
modernización de la Gestión Pública, el sistema de información y el gobierno electrónico, con el fin de mejorar la gestión pública al servicio del ciudadano. Si bien la modernización está en marcha, hay entidades que no pueden esperar, como son la mayoría de las gestiones municipales distritales $\mathrm{e}$ incluso la mayoría de municipalidades provinciales, donde se nota la carencia de sistemas automatizados de gestión, con todos los procedimientos tributarios, limitando el trabajo y el cumplimiento de su labor al funcionario público de turno, generando gestiones deficientes, con informes y reportes inexactos, contribuyentes con deudas desactualizadas y una morosidad alta, por falta de control en la administración tributaria.

Se observa, por los resultados reportados por el MEF, con el incumplimiento de metas en la gestión municipal, que están relacionadas con la aplicación deficiente, de las tecnologías de la información y la comunicación (TIC), generando contribuyentes morosos, tributos incobrables, recursos que no cubren el costo de los servicios que debe prestar la municipalidad.

Los antecedentes con variables similares relacionados a la investigación consideran como problema, la carencia de un sistema de información, generando a nivel administrativo limitaciones en la recaudación, con recursos económicos deficientes que no permiten dar el servicio que realmente corresponde.

Identifica (Campos, 2012), las consecuencias de la falta de recursos económicos que limitan cumplir con los servicios que debe brindar la municipalidad, causados por la evasión, morosidad y la falta de conciencia tributaria de los contribuyentes. En la organización interna, carecer de un sistema con información actualizada de los contribuyentes, hace deficiente, con un estado de iliquidez.

Pacheco y Torres (2012) sostienen que "para evaluar la variable del Proceso de Gestión Recaudadora del Impuesto Predial del SAT, según los diagnósticos, que el componente Información y Comunicación, que actualmente operan en la entidad, no facilita el proceso de recaudación del impuesto predial analizado, en tanto no generan información confiable ni transparente".

Por eso el objetivo de la investigación fue, determinar la manera en que influiría la aplicación de un Sistema Automatizado de Control de Valores Tributarios (SACVT) en la Gestión administrativa Tributaria; a su vez, identificar y analizar cómo influye el SACVT, tanto en la calidad de la información de las cuentas por cobrar y la recaudación tributaria en la municipalidad distrital. Sistema aplicable a la Administración Tributaria Municipal Distrital, que es una parte, de un todo de la Administración Pública, con funciones y facultades, normados por el estado y ejecutados en tres niveles de gobierno, como lo establece el Art. 189 (Constitución Política del Perú, 1993).

Para definir, la administración pública, partimos de lo que es Administración, según Chiavenato (2004) refiere que la administración es "el proceso de planear, organizar, dirigir y controlar el uso de los recursos para lograr los objetivos organizacionales"(p.8) y según Black y Porter (2006) ), definen a la administración como "el proceso de estructurar y utilizar conjuntos de recursos orientados hacia el logro de metas, para llevar a cabo las tareas en un entorno organizacional".(p.8)
Guerrero(2009) sostiene que "la administración pública y todos estos cambios se dan en la última década del siglo XX que apareció la nueva gerencia pública, una disciplina concebida en el seno de la economía neoclásica que fue propuesta como reemplazo de la administración pública, que sirve como fuente para los planes de modernización que se están dando en la gestión pública en general".

De acuerdo con Pareja (2006) ,señala que "las organizaciones públicas tienen las mismas necesidades que las organizaciones del sector privado por lo que respecta a alcanzar sus objetivos de una forma eficaz y eficiente, se acepta que sus zonas más operativas puedan y deban nutrirse de los conocimientos, herramientas y avances de la teoría administrativa”.(p.40)

Concluyendo con De la Encarnación( 2009), que define como un "conjunto de organismos que actúan bajo las órdenes del Poder Ejecutivo. Estos organismos son los encargados de dictar y aplicar las disposiciones necesarias para que se cumplan las leyes, fomentar los intereses públicos y resolver las reclamaciones de los ciudadanos". (p.2)

La administración pública en el Perú maneja los recursos de acuerdo con las normas establecidas, con el fin de servir al ciudadano. $\mathrm{Y}$ las normas de modernización del estado solo busca hacer gestiones eficientes en los tres niveles de gobierno. En la investigación realizada nos centramos solo en la Administración Tributaria de la municipalidad distrital, es decir gobierno local distrital, gestión encargada a la gerencia de administración tributaria o que es lo mismo gerencia de rentas, figura 1. 


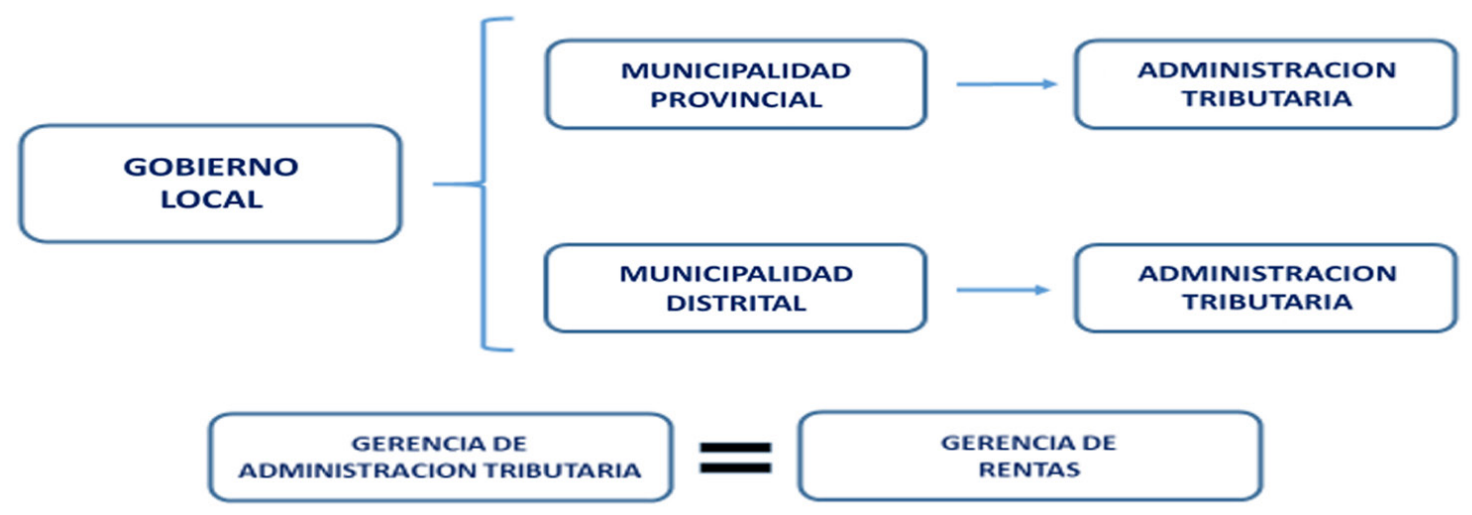

Figura 1. El Gobierno Local y la Administración Local. Fuente: Elaboración Propia.

La administración municipal, es parte de la administración pública y la potestad tributaria para poder ejercerlo está, en la Constitución Política del Perú, artículo 74, que tienen la posibilidad de crear, modificar, suprimir y exonerar dentro de su jurisdicción tasas y contribuciones, estando los arbitrios de limpieza pública, parque y jardines públicos y serenazgo dentro de la categoría tasas.

La Ley Orgánica de Municipalidades № 27972 artículo 5, 6 y 27, establece el marco, para la administración municipal y su organización, reconociendo al concejo municipal como ente normativo y fiscalizador, al alcalde como representante de la municipalidad y al gerente municipal como responsable administrativo. En el Art. 26, la Administración Municipal, adopta una estructura gerencial sustentándose en principios de programación, dirección, ejecución, supervisión, control concurrente y posterior.

El código tributario (D.S. $\mathrm{N}^{\circ} 133$ 2013-EF, 2013), define tres clases de tributos y uno de ellos es la tasa de Arbitrios, que son creados por las municipalidades con el instrumento normativo denominado "ordenanza", párrafo dos de la norma IV del título preliminar del Código Tributario, "los Gobiernos Locales, mediante Ordenanza, pueden crear, modificar y suprimir sus contribuciones, arbitrios, derechos y licencias o exonerar de ellos, dentro de su jurisdicción y con los límites que señala la Ley”.

Respecto a las funciones de la administración tributaria Municipal tenemos que están reglamentadas dentro del marco del Código Tributario (Decreto Supremo N 133-2013-EF, 2013) y la Ley de Tributación Municipal (Ley $\mathrm{N}^{\circ} 776,1993$ ). En el caso de la Municipalidad Distrital de San Luis, municipalidad fuente para la investigación, la administración tributaria, está a cargo la Gerencia de Rentas, órgano responsable y encargado de la recaudación de los tributos municipales, a través de los procesos cobranza y fiscalización tributaria. En ROF de la Municipalidad Distrital de San Luis (2006), ratifica las funciones exactas de la Gerencia de Rentas, como responsable de conducir, ejecutar los procesos administrativos, las políticas tributarias en forma eficiente y efecti- va, con el apoyo sus tres subgerencias, que son: la Subgerencia de Administración y Recaudación Tributaria, la Subgerencia de Fiscalización Tributaria y la Subgerencia de Cobranzas Coactivas. La recaudación tributaria inicia con la emisión anual de cuponeras, equivalente a la declaración y determinación del tributo (impuesto predial y la tasa de arbitrios) para el contribuyente.

Los tributos sirven para cumplir con los servicios a los ciudadanos de su jurisdicción y para fomentar la eficiencia de la gestión tributaria, el gobierno cada año dicta normas a través del ministerio de economía y finanzas, estableciendo metas de recaudación e implementación del sistema de administración tributaria, para ser sujetos de la transferencia de recursos económicos de parte del gobierno central a la municipalidad. Para ello, la gerencia de rentas administra los tributos, como el impuesto predial, las tasas de arbitrios (el barrido de calles, recojo de residuos sólidos, parques y jardines y serenazgo), que representan los principales recursos que recauda $y$ merecen la atención y control, a través del sistema automatizado. 


\section{LAS CUENTAS POR COBRAR Y LA RECAUDACIÓN TRIBUTARIA}

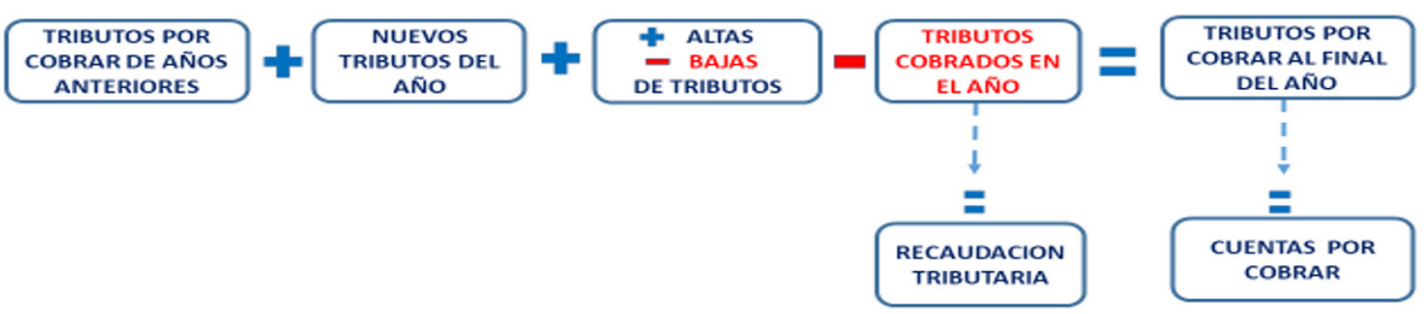

Figura 2. Las Cuentas por Cobrar de Tributos. Fuente: Elaboración Propia.

La determinación de las cuentas por cobrar de tributos es parte de la gestión tributaria, el cual es remitido al área de contabilidad, para formar parte de los estados financieros, en forma trimestral obligatoriamente, en cumplimiento al Reglamento de Organización y Funciones (ROF) de la municipalidad, y debe estar de acorde a las normas contables, es decir bajo los principios de integridad, uniformidad y al instructivo contable $\mathrm{n}^{\circ} 3$.

El informe de las cuentas por cobrar, es un reporte detallado de los movimientos de las cuentas por cobrar de tributos, de todos los contribuyentes, que suma el saldo inicial de tributos por cobrar de años anteriores, más la determinación o generación de nuevos tributos del año, más las altas de nuevos tributos determinados por la fiscalización o declaración voluntaria, menos la baja de tributos prescritos o que ya no corresponde y menos los tributos cobrados da como resultado al finalizar el año, las cuentas por cobrar de tributos (Figura 2). En la investigación se consideró solo los tributos (Impuestos y tasas por Cobrar) e ingresos no tributarios (Multas) que representan derechos exigibles a personas naturales y jurídicas.
La elaboración del informe de las cuentas por cobrar debe estar de acorde a las normas contable. La Ley No 28708 (2006) Ley General del Sistema Nacional de Contabilidad, resume los principios contables que son: Uniformidad, Integridad, Oportunidad, Transparencia, Legalidad. Las normas contables y sus modificaciones, aplicables a la gestión pública, así como los instructivos contables, las que sirven al órgano de control interno, como instrumento de medición, sobre la razonabilidad de los estados financieros y si se ajustan a los principio y normas contables respectivamente. Dichos estados financieros finalmente se consolidarán para elaborar las cuentas nacionales, de la Cuenta General de la República, su incumplimiento, genera sanciones para el funcionario responsable a través del Órgano de Control Interno (OCI), quien vela el uso eficiente, eficaz y económico de los recursos.

Según Claros y León (2012,), cita “al informe COSO que define al Control Interno, como un proceso efectuado por la junta directiva, la gerencia y otro personal designado, diseñado para proporcionar seguridad razonable respecto al logro de objetivos en las tres categorías siguiente: i) eficacia y eficiencia de las operaciones, ii) confiabilidad de la información financiera, y iii) cumplimiento con las leyes y regulaciones" (p.49).

Los sistemas automatizados completos están los del SAT de las municipalidades provinciales y el SIAF. Pero existe el SIAF-GL es el módulo de rentas que esta desde el 2010, pero no ha sido posible su implementación e instalación, incluso en el año 2011 fue una meta, pero las municipalidades no pudieron cumplirla, y no es aplicable para los SAT normativamente. Los sistemas más conocidos que usan las municipalidades, sin considerar las privadas son:

El SIAF-SP es un sistema de registro contable automatizado, de operaciones y procesamiento de la información de las transacciones que realiza el estado en sus 3 niveles de gobierno y su uso es obligatorio en el área contable, previos a la ejecución de proyectos y el abastecimiento de bienes y servicios de la entidad. El SIAF es considerado como una herramienta importante de la gestión financiera del tesoro público.(Alvarez, 2011,p.97) 
La Asociación de Servicios de Administración Tributario ASAT (2017), tiene solo 9 municipalidades provinciales, que cuentan con el sistema, que le permite realizar todo el proceso de administración, recaudación y control de los recursos municipales. Se sabe según el informe (ciudadanos al dia, 2004) Las SATs han logrado incrementar la recaudación e forma significativa. "Es importante impulsar el apoyo a la creación de instituciones similares dentro de los demás gobiernos locales para su gestión tanto a nivel de Lima, como a nivel nacional". El problema del SAT o el sistema que maneja, es inaplicable en las municipalidades distritales menos en una municipalidad de centro poblado, el modelo está dirigido para las municipalidades provinciales.

En la actualidad, existen municipalidades distritales de Lima, que tienen sus sistemas automatizados y su gestión así lo demuestra cómo es el caso de Miraflores, San Isidro, san Borja, Surco y la Molina, las que se pueden pagar los tributos en línea por vía internet. Y otros que lo hacen a través de una base de datos que tiene que ser actualizado diariamente con el banco. Que son las municipalidades que están con sistemas actuales pero incompletos y otros con sistemas obsoletos. Pero existen sistemas completos que están siendo usados por los Servicios de Administración Tributaria SAT, aplicables solo para las municipalidades provinciales, en la actualidad existen 196 municipalidades provinciales y según la Asociación de Servicios de Administración Tributaria (ASAT, 2017) las SAT que están funcionando a nivel nacional son 9, que representaría solo un 5\% del total.

La propuesta fue para la municipalidad distrital de San Luis, entidad con un sistema obsoleto con deficiencias que generan retrasos con la información de las cuentas por cobrar, para los usuarios internos, como contabilidad, planificación de las gerencias y las acciones propias de cobranzas. La gestión privada empresarial, utiliza sistemas en ERP, es decir, sistemas completos e integrados de gestión, aprovechando toda la tecnología de la información y de las comunicaciones, la realidad es que según (INEI Instituto Nacional de Estadistica e Informática, 2017) existen "1655 municipalidades distritales y provinciales, más 2,484 municipalidades de centros poblados", que esperan el plan bicentenario al 2021, cuya modernización está en marcha, pero es prematuro creer que si no llega a nivel distrital, menos aún será a los Centros Poblados.

Alcance

Determinar la influencia de un siste-

\section{Objetivo}

Se planteó determinar la manera en que influiría la aplicación del sistema, en la gestión administrativa tributaria de la Municipalidad Distrital de San Luis, distrito fuente de la investigación.

\section{MATERIAL Y MÉTODOS}

La investigación es cuantitativa de tipo descriptivo y explicativo, con resultados que ha permitido dilucidar la correlación entre las variables analizadas, Sistema Automatizado y la Gestión Administrativa Tributaria de la Municipalidad, confirmando que si influye la aplicación de un Sistema Automatizado de Control de Valores Tributarios (SACVT) en la Gestión administrativa Tributaria de la Municipalidad, constituyéndose un aporte válido y aplicable a otras municipalidades similares. ma automatizado en la Gestión Administrativa Tributaria de la Municipalidad Distrital.

\section{Diseño}

El Diseño fue no experimental, porque se realiza sin manipular las variables, ya que su propósito fue analizar e identificar de manera objetiva los datos de una realidad muestral de la gestión administrativa Tributaria, para después analizarlos; una investigación longitudinal que se mueve entre los años 2014 y 2015.

\section{Enfoque}

El enfoque es cuantitativo, porque se realizaron pruebas de correlación, probabilidad y mediciones con inferencia estadística.

\section{Unidad de Análisis}

La unidad de análisis y fuente de información fue, la Administración Tributaria de la Municipalidad Distrital de San Luis, que es representado en la municipalidad por la Gerencia de Rentas o Gerencia de Administración Tributaria.

\section{Datos y análisis de la Información}

Los datos de la investigación se recabaron de la población y muestra de estudio, de 23 funcionarios usuarios del sistema automatizado de la Administración Tributaria de la Municipalidad fuente, a través de una encuesta de 14 preguntas con tres alternativas cerradas, las que se adecuó a la escala de Likert y se aplicó la prueba $\mathrm{R}$ de Pearson, para validar la correlación de las variables y la probabilidad de error de la hipótesis de la investigación. 


\section{RESULTADOS}

La investigación confirmó la hipótesis, que "La aplicación de un Sistema Automatizado de Control de Valores Tributarios (SACVT) influiría directa y significativamente en la Gestión administrativa tributaria de la Municipalidad Distrital de San Luis". Donde la variable "Gestión Administrativa Tributaria" depende de la variable independiente "Sistema Automatizado de Control de Valores Tributarios (SACVT)", la misma que fue propuesto como una solución al problema. El muestreo fue cualitativo intencionado y la población se ajustó finalmente al $100 \%$ para el muestreo analizado. La prueba se realizó a 23 funcionarios y servidores públicos, usuarios del sistema y que laboraron con la gerencia de rentas en los años 2014 y 2015, o laboran en la Municipalidad Distrital de San Luis, fuente de la investigación. Se aplicó la encuesta con 14 preguntas, considerando indicadores que reflejen, eficiencia de la gestión, las limitaciones del sistema, la calidad de información para el usuario interno o ciudadano. Concluyendo con la revisión y análisis documentario de los instrumentos de gestión, memorias anuales del 2014 y 2015. Que están disponibles por transparencia.

En la Tabla 1, se muestra las respuestas de eficiente, regular y deficiente de la "Gestión Administrativa Tributaria" hay un $8,7 \%$ considera totalmente deficiente y entre regular más deficiente $60,9 \%$ y solo un $39,1 \%$ considera eficiente la gestión, el mismo resultado se da enla figura 4 y con relación al "Sistema Automatizado de Control de Valores Tributarios (SACVT), el resultado fue, $39,1 \%$ totalmente deficiente y entre regular más deficiente $69.5 \%$ y solo un $30.4 \%$ considera eficiente el sistema, como se visualiza en la figura 5, confirmando las limitaciones del sistema y con una gestión administrativa tributaria deficiente.

Tabla 1. Gestión Administrativa Tributaria.

Fuente: SPSS - Data1.Sav - Elaboración Propia.

\begin{tabular}{lcccc} 
& Frecuencia & Porcentaje & Porcentaje válido & Porcentaje acumulado \\
Inadecuada o deficiente & 2 & $8,7 \%$ & $8,7 \%$ & $8,7 \%$ \\
\hline Regular & 12 & $52,2 \%$ & $52,2 \%$ & $60,9 \%$ \\
\hline Adecuada o eficiente & 9 & $39,1 \%$ & $39,1 \%$ & $100,0 \%$ \\
\hline Total & 23 & $100,0 \%$ & $100,0 \%$ & \\
\hline
\end{tabular}

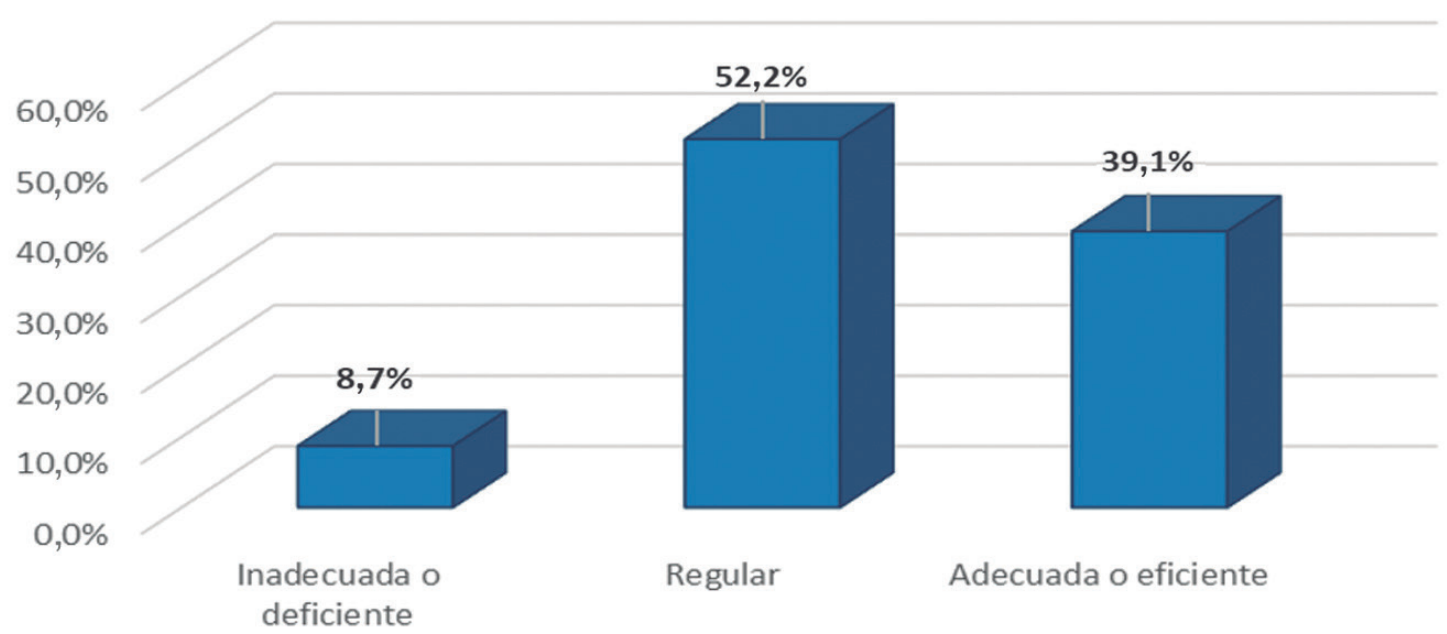

Figura 3. Gestión Administrativa Tributaria.

Fuente: SPSS - Data1.sav - Elaboración propia. 
Tabla 2. Sistema Automatizado de Control de Valores Tributarios (SACVT). Fuente: SPSS - Data1.Sav - Elaboración Propia.

\begin{tabular}{|c|c|c|c|c|}
\hline & Frecuencia & Porcentaje & Porcentaje válido & Porcentaje acumulado \\
\hline Inadecuada o deficiente & 9 & $39,1 \%$ & $39,1 \%$ & $39,1 \%$ \\
\hline Regular & 7 & $30,4 \%$ & $30,4 \%$ & $69,6 \%$ \\
\hline Adecuada o eficiente & 7 & $30,4 \%$ & $30,4 \%$ & $100,0 \%$ \\
\hline Total & 23 & $100,0 \%$ & $100,0 \%$ & \\
\hline
\end{tabular}

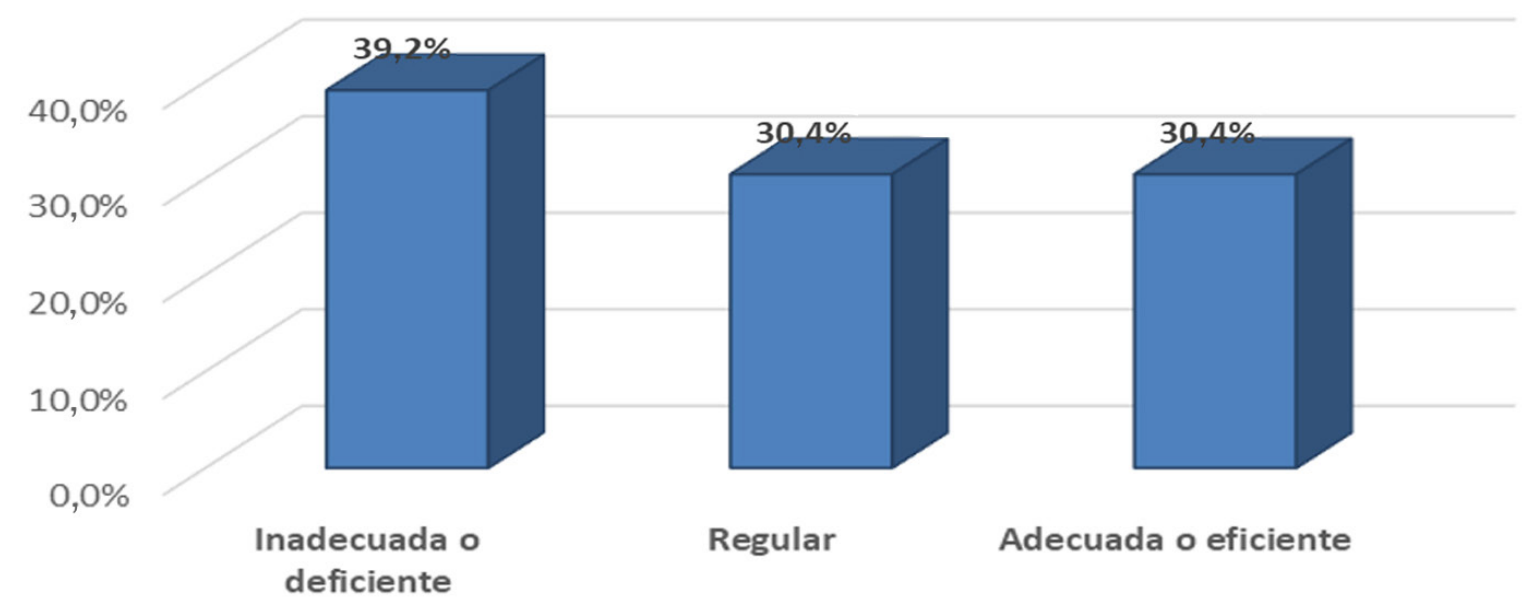

Figura 4. Automatizado de Control de Valores Tributarios.

Fuente: SPSS - Data1.sav - Elaboración propia.

Tabla 3. $R$ de Pearson - Sistema Automatizado de Control

de Valores Tributarios (SACVT) \& Gestión

Administrativa Tributaria.

Fuente: SPSS - Data1.sav - Elaboración propia.

\begin{tabular}{ccc} 
& $\begin{array}{c}\text { Estadísticos para una muestra } \\
\text { Sistema Automatizado de Control } \\
\text { de Valores Tributarios }\end{array}$ & $\begin{array}{c}\text { Gestión Administrativa } \\
\text { Tributaria }\end{array}$ \\
Correlación de Pearson & 1 & 0,981 \\
\hline $\begin{array}{c}\text { Sig. (bilateral) } \\
\text { productos cruzados }\end{array}$ & 2,000 & 0,019 \\
\hline Covarianza & 0,034 & $-1,000$ \\
\hline $\mathrm{N}=$ muestra & 23 & 0,026 \\
\hline
\end{tabular}

${ }^{*}$ La Comparación es significativa al nivel 0,05 (unilateral). 


\section{DISCUSIÓN}

Mientras el Gobierno Central evidencia la problemática, porque en el informe anual, 2016-2017 de la Comisión del Congreso, dice; que los Gobiernos locales y Modernización de la Gestión del Estado, en su evaluación indica que: "Estamos lejos de tener un Estado moderno. Modernizar el Estado implica mejorar la Gestión Pública”. Lo mismo dice sobre Gobierno Digital: "Se presenta un limitado avance en gobierno digital. Los ciudadanos no cuentan con herramientas tecnológicas en línea que hagan posible una relación con las distintas dependencias nacionales, regionales o locales que les permitan acercar el Estado a las personas y disminuir los costos de transacción”. Los resultados de la investigación confirman la problemática y abre un camino para dar solución, a los sistemas automatizados de la Administración Tributaria de las municipalidades.

El incumplimiento de metas establecidas por el MEF para las municipalidades, evidencian dentro de la administración tributaria, que no se han cumplido con disponer la información en línea a través de las páginas web de la municipalidad y no se ha llegado a recaudar los tributos necesarios establecidos para el año. Así como existen estudios como de, Guerrero, (2011), quien manifiesta que varias causas que originan la debilidad de la estructura tributaria como fuente de ingresos propios, entre las cuales se puede detectar problemas similares en el proyecto actual, como que la cobranza se efectúa sin control real y concentrando esfuerzos en cobrar las cuentas de más alto grado o monto; es decir por la falta de herramientas que permitan llevar el control al 100\%. Que están relacionados a mejorar la recaudación, tener la información de los tributos por cobrar, para culminar con el proceso de cobranza y el manejo de cuadros de control, todos para mejorar la Gestión Tributaria, pero por falta de decisión política de la municipalidad, muchos continúan con el problema. Se debe admitir que existen municipalidades con gestiones eficientes, por dos fortalezas básicas que tomaron la decisión de implementar su administración tributaria y tienen mejores recursos económicos.

Según los resultados de la investigación, el sistema de la Administración Tributaria tiene limitaciones, y requiere de su implementación de un sistema automatizado con todos los procedimientos de cobranza. Esto permitiría facilitar el trabajo y corregir las deficiencias observadas en la Gestión Administrativa Tributaria de la Municipalidad, el cual tiene solución gradual y aplicable a corto plazo.

La implementación de un sistema automatizado en la Administración Tributaria permitiría cumplir con las políticas de modernización del estado, es decir cumplir con las metas establecidas por el gobierno central a través del MEF. Consecuentemente permitiría a la administración tener la información oportuna para la toma de decisiones y la administración y el control de los valores tributarios, generaría una mayor recaudación tributaria.

El estado está en proceso de modernización, pero la realidad es que aún no llega a la mayoría de las municipalidades distritales, menos a las municipalidades de centros poblados, la investigación ha demostrado que es una necesidad su implementación y que si es viable su ejecución aplicación.

\section{REFERENCIAS BIBLIOGRÁFICAS}

Alvarez, J. (2011). Sistema de Información de Administración Financiera - Resumen General. Lima: Instituto Pacifico SAC.

ASAT. (2017). Asociación de Servicios de Administración Tributaria. Trujillo. Obtenido de http://www. asat.org.pe/index.php/asat/directorio-electronico.

Campos, B. (2012). Las Deudas de tributos y sus efectos en la Liquidez de la Municipalidad Distrital de Paijan Periodo 2010-2011. Trujillo: Universidad Nacional de Trujillo.

Chiavenato, I. (2004). Introducción a la Teoría General de la Administración. Séptima Edición. México. D.F.: McGraw-Hill Interamericana.

De la Encarnación, M. (2009). Administración Pública - Administración $y$ finanzas. Segunda Edición. Madrid: Ediciones Paraninfo.

Castro , D. (2015). Ley Orgánica de Municipalidades - No 27972 - Concordada y Comentada. Tercera Edición. Lima: Ubi lex Asesores.

Black, H. \& Porter, G. (2006). Administración. Novena Edición, Mexico D.F. Pearson Educación.

INEI Instituto Nacional de Estadistica e Informática. (Enero de 2017). Estadisticas Municipales 2016. Obtenido de https://www.inei.gob.pe/ media/MenuRecursivo/publicaciones_digitales/ Est/Lib1417/libro. pdf.

Ley $\mathrm{N}^{\circ}$ 776. (1993). Ley De Tributación Municipal. Lima. Diario El Peruano. 
Pacheco M. \& Torres Y. (2012). Sistema de Control Interno para el Proceso de Gestión Recaudadora del Impuesto Predial del Servicio de Administración Tributaria de Lima en el 2012. Lima:Universidad Nacional de Ingenieria.
Pareja, L. (2006). Contabilidad Gubernamental I. Lima. Departamento de Impresiones y Publicaciones UIGV.

Claros R. \& León ,O. (2012). El Control Interno Como Herramienta de Gestión y Evaluación. Primera Edi- ción. Lima. Pacífico Editores S.A.C.

SAT Servicio de Administración Tributaria de Lima. (2009). Guia Para la Elaboración de Costos de los Servicios Públicos en el Perú. Lima. AFA Editores Importadores S. A 